\title{
DORMICIÓN FÍSICA EN LEPECHINIA FLORIBUNDA (LAMIACEAE): UN ARBUSTO NATIVO DE INTERÉS MEDICINAL
}

\author{
LORENA ASHWORTH ${ }^{1 *}$, JULIA CAMINA ${ }^{1}$ y GUILLERMO FUNES 1,2
}

\begin{abstract}
Summary: Physical seed dormancy in Lepechinia floribunda (Lamiaceae): a medicinal native shrub. Lepechinia floribunda is an aromatic and medicinal shrub with multiple uses. The low germination of its seeds is the main limiting trait for propagation. The goal of this study was to test the presence of physical seed dormancy in L. floribunda and to assess the effectiveness of time of storage and different pregermination treatments in order to overcome such dormancy. We conducted an imbibition experiment and tested germination in seeds stored for 0,90, 270, and 1050 days, which were subjected to pretreatments of thermal (water at $100^{\circ} \mathrm{C}$ ) and chemical scarification (sulphuric acid). Seeds of $L$. floribunda have physical dormancy, and the most effective treatment to break it was sulphuric acid, surpassing $70 \%$ of germination. Time of storage did not affect the state of dormancy, and the viability of seeds was maintained high for at least 3 years. Results from this study will help to establish strategies of sexual propagation of this native useful plant species.
\end{abstract}

Key words: Chemical scarification, Lamiaceae, Lepechinia floribunda, seed dormancy, seed germination, seed imbibition.

Resumen: Lepechinia floribunda es un arbusto aromático medicinal que tiene múltiples aplicaciones. La baja germinación de sus semillas es el principal rasgo que hasta el momento ha limitado su propagación. El objetivo del presente trabajo fue determinar la presencia de dormición física en semillas de $L$. floribunda y evaluar la eficiencia de distintos pretratamientos y el tiempo de almacenamiento en romper dicha dormición. Se realizó un experimento de imbibición y otros de germinación con semillas almacenadas por $0,90,270$ y 1050 días utilizando escarificación térmica (con agua a $100^{\circ} \mathrm{C}$ ) y química (ácido sulfúrico concentrado) como pretratamientos. Las semillas de L. floribunda presentaron dormición física y el tratamiento más efectivo para romper la dormición fue el ácido sulfúrico, con valores que superan el $70 \%$ de germinación. El tiempo de almacenamiento no afectó el estado de dormición y la viabilidad de las semillas se mantuvo alta durante 3 años. Estos resultados ayudarán a establecer estrategias de propagación sexual para esta especie nativa de gran interés aplicado.

Palabras clave: Escarificación química, Lamiaceae, Lepechinia floribunda, dormición, germinación de semillas, imbibición.

\section{INTRODUCCIÓN}

Las especies vegetales medicinales se caracterizan por la producción de compuestos químicos derivados del metabolismo secundario que son de gran interés en la industria farmacéutica,

\footnotetext{
${ }^{1}$ Instituto Multidisciplinario de Biología Vegetal, Consejo Nacional de Investigaciones Científicas y Técnicas (CONICET), Universidad Nacional de Córdoba (UNC), Argentina. C.C. 495, (5000) Córdoba, Argentina. Tel. (54) 351-433-1056/2104. ${ }^{2}$ Facultad de Ciencias Exactas, Físicas y Naturales, UNC, Argentina. *loashworth@ gmail.com
}

agronómica, alimentaria y cosmética, entre otras (Barboza et al., 2009; Chen et al., 2016). A nivel mundial, la gran demanda de plantas medicinales es suplida en su gran mayoría por cosechas casi exclusivamente extractivas y sólo existen contados casos donde son cultivadas (Chen et al., 2016; Vasisht et al., 2016). Algunos parámetros que ilustran la magnitud de la extracción que están soportando las poblaciones silvestres, es el volumen de consumo y utilización de plantas medicinales a nivel mundial. Anualmente se comercializan más de 700 mil toneladas de plantas medicinales y el valor de su comercialización supera los 3 mil millones de dólares (Vasisht et al., 2016). Como 
consecuencia de la creciente demanda, de las políticas de mercado regional e internacional, la falta de control y regulación y la pérdida de hábitats naturales, casi la mitad de las especies medicinales en el mundo se encuentran en riesgo de extinción (IUCN, 2007; Chen et al., 2016).

Entre las múltiples soluciones a esta problemática, la más recomendada por organismos internacionales dedicados a la conservación de la biodiversidad, es el reemplazo de la cosecha de poblaciones silvestres, por el cultivo de plantas medicinales (Schippman et al., 2002). Notablemente, muchas veces los aspectos que impiden avanzar sobre la posibilidad de cultivo de estas especies es la falta de conocimiento sobre requerimientos específicos de su propagación (Shippman et al., 2002). La gran familia cosmopolita Lamiaceae, es reconocida mundialmente por su gran importancia económica, sin embargo, los requerimientos de propagación han sido estudiados sólo en algunos géneros (Baskin \& Baskin, 2014). En particular muy poco se sabe sobre los requerimientos germinativos del género Lepechinia Willd, que se distribuye únicamente en el continente Americano, habitando zonas andinas y montañosas principalmente en Sudamérica (Drew \& Sytsma, 2013).

Los resultados reportados hasta el momento para Lepechinia chamaedryoides, endémica de Chile y L. floribunda, nativa de Argentina, sugieren la existencia de algún tipo de dormición vinculada con la impermeabilidad de la cubierta seminal (Aráoz et al., 2011; Serri et al., 2011). En Argentina coexisten tres especies Lepechinia floribunda, L. meyenii y L. vesiculosa (Drew \& Sytsma, 2013; O’leary 2015). L. floribunda (Benth.) Epling es un arbusto aromático de flores blanco-azuladas que produce semillas negras pequeñas $(2.6-2.8 \mathrm{~mm}$, Scandalaris y Barboza, 2013) y livianas (5 $\pm 0,6$ $\mathrm{mg}$ ), que se desprenden fácilmente de los frutos a la madurez y su dispersión ocurre por gravedad (L.A. Obs. pers). L. floribunda tiene múltiples usos, descongestiva (Arias Toledo el al., 2010), antiviral (Duschatzky et al., 2007) y antimicrobiana, entre otros (Palacios et al., 2007). A pesar de esta variedad de usos, información sobre la propagación de esta especie es muy escasa e indica que los porcentajes de germinación de semillas son generalmente muy bajos y erráticos, lo que ha dificultado su propagación sexual (Aráoz et al., 2011). Aráoz et al. (2011) obtuvieron porcentajes de germinación muy bajos en semillas donde aplicaron tratamientos para romper la dormición fisiológica (e.g. GA3, estratificación en frío), esto sugiere que las semillas de esta especie podrían presentar otro tipo de dormición, posiblemente dormición física como sugieren los resultados de Serri et al. (2011) para L. chamaedryoides. Asimismo, la dormición física esta dada por la presencia de una o varias capas de células impermeables al agua en la cubierta de las semillas, lo que impide la imbibición de la misma (Baskin \& Baskin, 2014). En un estudio morfoanatómico de las semillas de L. floribunda se observó la presencia en el mesocarpo, de una o dos capas de células parenquimáticas en empalizada (23-40 $\mu \mathrm{m}$, Scandalaris \& Barboza, 2013) que podrían ser las responsables de la impermeabilidad de la cubierta seminal. Para superar este tipo de dormición es necesario realizar tratamientos pregerminativos que permitan romper dicha impermeabilidad (Baskin \& Baskin, 2014). Finalmente, otro aspecto de importancia al momento de generar protocolos de conservación y propagación de especies de interés es conocer si el tiempo de almacenamiento afecta el estado de dormición de las semillas (Hong \& Ellis, 1996).

Los objetivos de este estudio fueron determinar la presencia de dormición física y la eficiencia de tres métodos, escarificación química (ácido sulfúrico concentrado) y térmica (agua a $100^{\circ} \mathrm{C}$ ) y tiempo de almacenamiento, para la salida de este estado en L. floribunda. Para concretar estos objetivos realizamos un experimento de imbibición de semillas y dos experimentos manipulando el tiempo de almacenamiento de las semillas y aplicando tratamientos pregerminativos de escarificación química y térmica que permitan romper la impermeabilidad de la cubierta seminal (Baskin \& Baskin, 2014). La información generada en este estudio es de particular importancia para la elaboración de protocolos de conservación y propagación de L. floribunda y podría ser aplicable a otras especies de este género con dormición física.

\section{Material y Métodos}

En diciembre de 2010 se recolectaron semillas (núculas) de L. floribunda en cuatro sitios de Bosque Chaqueño Serrano de la provincia de Córdoba, Argentina, donde la especie crece 


\section{Ashworth et al. - Dormición física en semillas en Lepechinia floribunda}

naturalmente (Giorgis et al., 2010). Los sitios fueron seleccionados por presentar similitudes en condiciones climáticas, altitudinales y composición de especies. En total se colectaron semillas de 80 plantas, distribuidas en los siguientes sitios: Reserva Natural Los Manantiales (31 99'40.34" S, 6421'03.67" W), Reserva Hídrica La Quebrada (31 ${ }^{\circ}$ '11.29” S, 64²0'28.85” W), Estancia privada Santo Domingo (31 ${ }^{\circ} 10^{\prime} 57.09^{\prime \prime} \mathrm{S}, 64^{\circ} 15^{\prime} 40.05^{\prime \prime}$ W) y Camino al Cuadrado $\left(31^{\circ} 7^{\prime} 53.08^{\prime \prime} \mathrm{S}\right.$, 64¹9'53.04" O). Las semillas fueron procesadas en laboratorio, las sanas fueron almacenadas y las que presentaban signos de daño por depredadores fueron descartadas. Considerando que en estudios previos con semillas de esta especie se observó la existencia de una cubierta de células que podrían ser impermeables al agua (Scandalaris \& Barboza, 2013), es que se asumió que las semillas de todos los sitios tendrían dormición física y por lo tanto las semillas de los diferentes sitios fueron agrupadas en un único pool. Las semillas fueron almacenadas en bolsas de papel, en oscuridad y a temperatura ambiente $\left(25-28^{\circ} \mathrm{C}\right)$ y este pool fue utilizado para evaluar los métodos de salida de la dormición. Asimismo, con el fin de corroborar la presencia de dormición también se realizó una curva de imbibición. Para tal fin en diciembre de 2015 se colectaron semillas de L. floribunda en los mismos sitios y se procesaron y almacenaron siguiendo la metodología antes mencionada, estas semillas fueron utilizadas para el experimento de imbibición realizado en enero de 2016.

\section{Experimentos de germinación}

Para cada experimento se realizaron tres réplicas (cápsula de Petri de $9 \mathrm{~cm}$ de diámetro con una base de papel de filtro) y en cada cápsula se colocaron 30 semillas que fueron regadas con agua destilada y colocadas en cámaras de germinación en condiciones controladas de luz (fotoperíodo) y temperatura (termoperíodo). El fotoperíodo fue generado con tubos de luz blanca fría fluorescente (38 $\left.\mu \mathrm{mol} \mathrm{m} \mathrm{m}^{-2} \mathrm{~s}^{-1}\right)$. Todos los experimentos se desarrollaron durante 30 días y en todos los casos el mayor valor de temperatura coincidió con las horas de luz. En todos los experimentos las condiciones de germinación fueron $25 / 15^{\circ} \mathrm{C}$ (12 h fotoperíodo). El régimen de temperatura seleccionado corresponde al óptimo para la germinación de la mayoría de las especies del Bosque Chaqueño Serrano (Funes et al., 2009). El número de semillas germinadas se registró diariamente y las semillas se consideraron germinadas al presentar radículas de $2 \mathrm{~mm}$ de largo.

\section{Experimento de imbibición}

El experimento de inhibición consistió en dos tratamientos, semillas escarificadas y no escarificadas. En el tratamiento de escarificación las semillas fueron sumergidas en $\mathrm{H}_{2} \mathrm{SO}_{4}$ concentrado por 30 minutos y posteriormente lavadas bajo una corriente de agua por dos minutos para eliminar los restos de ácido. En el tratamiento control las semillas no recibieron ningún tratamiento. Las semillas fueron colocadas en cápsulas de Petri de $9 \mathrm{~cm}$ de diámetro con una base de papel de filtro humedecido con agua destilada y las cápsulas fueron colocadas en cámara de germinación. Luego de 0, 1, 2, 4, 7 y $24 \mathrm{~h}$, la superficie de las semillas fue secada y el pool de semillas de cada cápsula fue pesado en balanza de precisión $(0.1$ $\mathrm{mg}$ ) y regresado a las cápsulas de Petri (Susko et al., 2001). La masa de las semillas al comienzo del experimento (tiempo 0) fue el valor de referencia utilizado para evaluar el ingreso de agua a lo largo del tiempo. La cantidad de agua absorbida por las semillas fue cuantificada como el incremento en la masa y convertida a porcentaje (Baskin \& Baskin, 2014). Luego de estas mediciones y transcurridos 30 días se registró el porcentaje de germinación.

Experimento de escarificación química y tiempo de almacenamiento

La escarificación de semillas con ácido sulfúrico $\left(\mathrm{H}_{2} \mathrm{SO}_{4}\right)$ concentrado y el tiempo de almacenamiento son técnicas que han demostrado ser efectivas para romper la dormición de las semillas (Baskin \& Baskin, 2014). Para evaluar el efecto de ambas técnicas sobre la germinación, las semillas fueron almacenadas durante $0,90,270 \mathrm{y}$ 1095 días (3 años) y sometidas a un pretratamiento con ácido sulfúrico concentrado durante 30 minutos y posterior lavado de las semillas durante dos minutos con agua corriente. Además, para los cuatro tiempos de almacenamiento también se consideró un tratamiento control donde las semillas no recibieron pretratamiento con ácido.

\section{Experimento de escarificación térmica}

Semillas almacenadas durante 0 y 90 días recibieron un pretratamiento con agua a $100^{\circ} \mathrm{C}$ 
durante 1,5 y 10 minutos y un grupo control que no recibió pretratamiento. Las semillas fueron colocadas en cápsulas de Petri y en cámaras de germinación.

\section{Análisis estadísticos}

Todos los análisis estadísticos fueron realizados con el programa R (R Core Team, 2014, versión 3.1.1). Se realizaron modelos lineales generalizados (GLM), para lo cual se usó la función $\mathrm{glm}$ del paquete MASS (Venables \& Ripley, 2002). Al evaluar el efecto de la escarificación química sobre el incremento de la masa de las semillas se utilizó distribución normal del error y al evaluar el efecto de los pretratamientos y el tiempo de almacenamiento sobre el porcentaje de germinación se utilizó distribución cuasibinomial del error (debido a la existencia de sobredispersión). La significancia de los factores y de la interacción se determinó comparando dos modelos mediante pruebas Chi cuadrado, un modelo con el factor o la interacción de interés y el otro modelo sin dicho factor. Diferencias entre niveles de un factor (control sin escarificación y escarificación con ácido sulfúrico) se evaluaron mediante la prueba $t$.

\section{Resultados}

\section{Experimento de imbibición}

Luego de $24 \mathrm{~h} \mathrm{a} 25 / 15^{\circ} \mathrm{C}$, el $100 \%$ de las semillas escarificadas se embebió, incrementando su masa en un $105 \%$ en promedio. De las semillas no escarificadas sólo el $6,67 \%$ se embebió y esto dio como resultado un incremento en masa del 9,5\% en promedio (Fig. 1). El incremento en la masa de las semillas fue significativamente mayor en las escarificadas que en las no escarificadas $(F=197, P=0,00015$, Fig. 1). Similarmente, luego de un mes, el porcentaje de germinación fue significativamente mayor $(F=34,7, P=0,004)$ en las semillas escarificadas $(76,44 \pm 16 \%)$ que en las no escarificadas $(12,22 \pm 2 \%)$. Cabe destacar que de las semillas no escarificadas que no germinaron (aproximadamente $88 \%$ ), ninguna presentó síntomas de imbibición (e.g. aumento de tamaño).

\section{Experimento de escarificación química y tiempo de almacenamiento}

La interacción entre tiempo de almacenamiento y pretratamiento con ácido sulfúrico no fue significativa $(F=2,03, P=0,17)$. El pretratamiento con ácido sulfúrico incrementó dieciséis veces la germinación, alcanzando valores superiores al $70 \%$, mostrando diferencias significativas respecto al control $(F=78,90, P<0,001$, Fig. 2). Por el contrario, el tiempo de almacenamiento fue un factor que no afectó significativamente el porcentaje de germinación de las semillas $(F=0,20$, $P=0,82$, Fig. 2).

\section{Experimento de escarificación térmica}

La germinación de semillas almacenadas por 0 y 90 días que recibieron pretratamiento con agua a $100^{\circ} \mathrm{C}$ durante 1,5 y 10 minutos fue cero, mientras que en el tratamiento control la germinación fue de $3,5 \pm 2 \%$.

\section{Discusión}

El resultado más importante de este estudio revela que los bajos porcentajes de germinación en semillas no escarificadas de L. floribunda son producto de dormición física. Las semillas de L. floribunda tienen una cubierta que impide el ingreso de agua. Esta impermeabilidad estaría dada por las de células del parénquima en empalizada (Baskin et al., 2000) que se encuentran formando una o dos capas de 23-40 $\mu \mathrm{m}$ en el mesocarpo de sus semillas (Scandalaris \& Barboza, 2013). De las técnicas evaluadas en este estudio, la única que permitió romper dicha impermeabilidad y alcanzar altos porcentajes de germinación fue la escarificación química, no así la escarificación térmica ni el tiempo de almacenamiento. La comparación de nuestros resultados con los obtenidos para L. chamaedryoides usando ácido sulfúrico diluido durante 30 minutos, sin efectos sobre la germinación (Serri et al., 2011), indicaría que la concentración del ácido es un aspecto clave.

Es interesante destacar que la impermeabilidad y dormición de las semillas de L. floribunda no fue del $100 \%$ ya que en los tratamientos control de cada experimento un porcentaje bajo de semillas se embebieron y germinaron. Esta variabilidad en la impermeabilidad de las semillas es un rasgo que ya se ha observado en otras especies con dormición física (e.g. Susko et al., 2001; Galíndez et al., 2015), incluso en L. chamaedryoides donde hubo cierto porcentaje de imbibición y germinación en semillas 


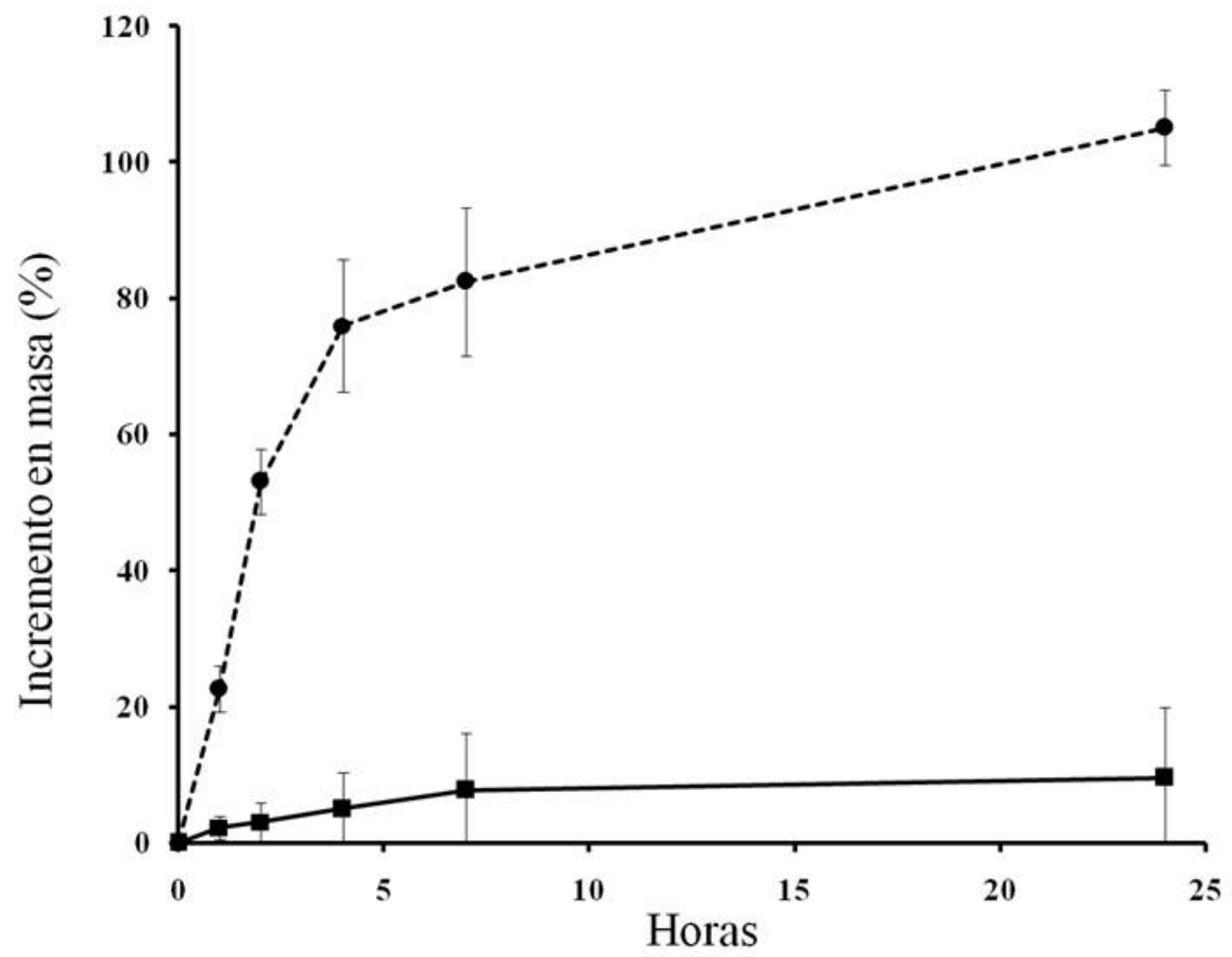

Fig. 1. Media \pm desvío estándar del porcentaje de incremento en la masa de semillas de Lepechinia floribunda luego de $24 \mathrm{hs}$ en papel de filtro húmedo y a $25 / 15^{\circ} \mathrm{C}$. Con línea de puntos se muestra el tratamiento de escarificación de las semillas con ácido sulfúrico y con línea continua el tratamiento control (semillas no escarificadas).

que no recibieron pretratamiento (Serri et al., 2011). El diseño experimental de nuestro estudio no nos permitió evaluar factores involucrados en la variabilidad de la dormición, es decir, si dicha variabilidad es explicada mayormente a escala de individuos o poblaciones. Sin embargo, con base en los resultados aquí obtenidos sería interesante evaluar en futuros estudios cómo se distribuye esta variabilidad. La amplia distribución latitudinal y altitudinal que presenta esta especie permitiría evaluar además la relación entre los niveles de dormición y dichos gradientes ambientales.

Otro resultado importante es que las semillas de L. floribunda mantuvieron su viabilidad al menos por tres años que fue el mayor tiempo de almacenamiento evaluado en este trabajo. De esta manera se pone en evidencia que las semillas de L. floribunda además de presentar dormición física mantienen una alta viabilidad temporal, características que le permitirían formar bancos de semillas en el suelo (Susko et al., 2001; Baskin \& Baskin, 2014). La formación de bancos de semillas es un rasgo que podría contribuir al mantenimiento y resiliencia de las poblaciones naturales frente a disturbios antrópicos y eventos extremos producto del cambio climático (Hudson et al., 2015).

En función de nuestros hallazgos y con base en evidencia disponible para otros géneros de la familia sería importante evaluar técnicas alternativas para romper la dormición de las semillas, ya que 


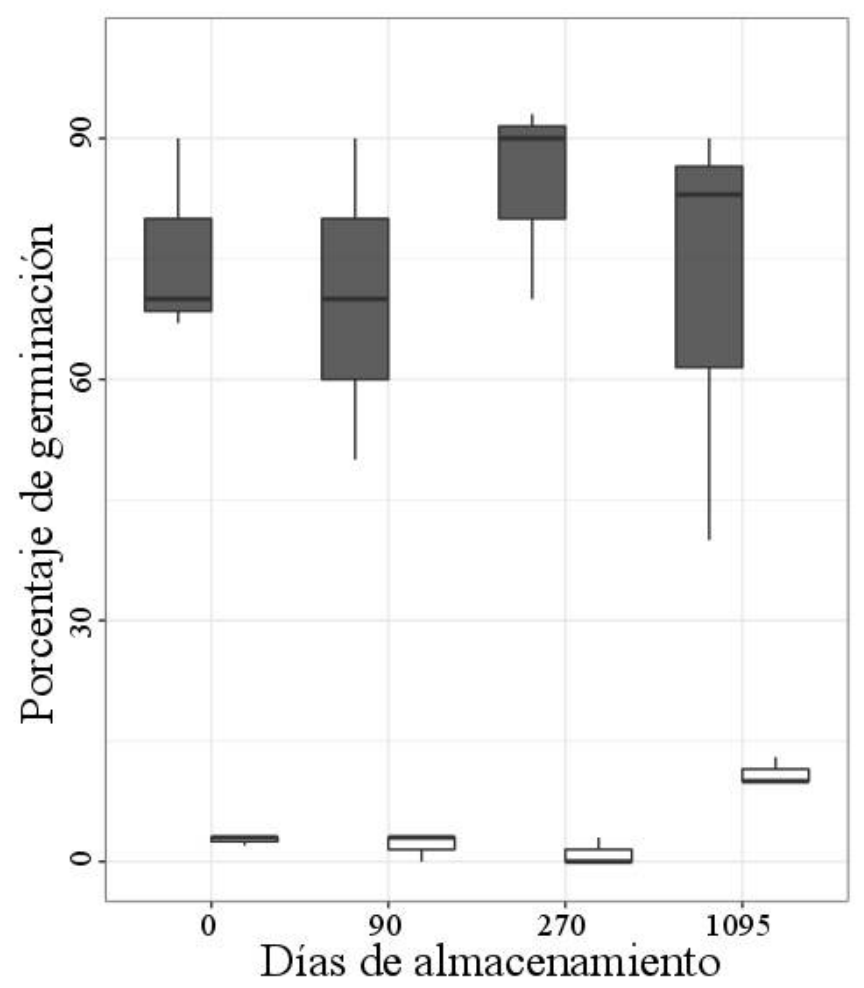

Fig. 2. Porcentaje de germinación de semillas de Lepechinia floribunda almacenadas durante 0 , 90, 270 y 1095 días a temperatura ambiente y sometidas a pretratamiento de escarificación con ácido sulfúrico concentrado durante 30 minutos (cajas negras) y control, semillas no escarificadas (cajas blancas). El límite inferior y superior de cada caja es el percentil 25 y 75 respectivamente. Las líneas horizontales corresponden a las medianas y las líneas verticales indican 1.5 veces el rango intercuartílico (Prueba $t, P<0,05$ ).

la escarificación con ácido requiere condiciones especiales para la manipulación. En particular sería importante evaluar qué factores son los responsables de romper la dormición de las semillas en condiciones naturales. En este sentido, se ha demostrado que el fuego es un factor que puede inducir la germinación en algunas especies de Lamiaceae (Catav et al., 2014). Aún cuando no se han realizado estudios en especies de Lepechinia, el hecho de que luego de un incendio las poblaciones de algunas especies de este género se expandan (Drew \& Sytsma, 2013), podría relacionarse con esta característica de la familia. Por lo tanto, en futuros estudios se debería evaluar el efecto del fuego y el humo en la germinación de esta especie. El conocimiento aquí generado aporta valiosa información sobre la germinación y dormición de las semillas de L. floribunda y seguramente será de gran utilidad para proyectos orientados a establecer estrategias de conservación y propagación de especies de interés medicinal.

En síntesis, las semillas de L. floribunda presentan dormición física y una alta capacidad germinativa. La escarificación química con ácido sulfúrico concentrado incrementó significativamente la germinación de sus semillas, no así la escarificación térmica, donde no hubo germinación. La viabilidad de las semillas almacenadas a temperatura ambiente por tres años fue alta y el tiempo de almacenamiento no afectó el estado de dormición.

\section{Agradecimientos}

Al Consejo Nacional de Investigaciones Científicas y Técnicas (CONICET) y a la 


\section{Ashworth et al. - Dormición física en semillas en Lepechinia floribunda}

Universidad Nacional de Córdoba (UNC) por las facilidades brindadas para el desarrollo de este estudio, a dos revisores anónimos por los valiosos aportes realizados a este trabajo y a las autoridades de las Reservas Los Manantiales y La Quebrada y propietarios de Estancia Santo Domingo por los permisos concedidos para trabajar en dichos lugares. Este estudio fue financiado por la Agencia Nacional de Promoción Científica y Tecnológica (Pict 1606-2011) y por el CONICET (PIP 03712015). L.A. y G.F. son investigadores de CONICET y J.L.C. es alumna de doctorado de la F.C.E.F y N de la UNC.

\section{Biblografía}

ARÁOZ, S., C. EYNARD \& D. PERAZZOLO. 2011. Estudios preliminares sobre calidad de semillas de Lepechinia floribunda (Benth.) para su domesticación. Análisis de semillas 20: 45-49.

ARIAS TOLEDO, B., C. TRILLO \& M. GRILLI. 2010. Uso de plantas medicinales en relación al estado de conservación del bosque en Córdoba, Argentina. Ecol. Austral 20: 235-246.

BARBOZA, G. E., J. J. CANTERO, C. NÚÑEZ, A. PACCIARONI \& L. ARIZA-ESPINAR. 2009. Medicinal plants: A general review and a phytochemical and ethnopharmacological screening of the native Argentine flora. Kurtziana 34: 7-365.

BASKIN, J.M., C.C. BASKIN \& X. LI. 2000. Taxonomy, anatomy and evolution of physical dormancy in seeds. Plant Sp. Biol. 15: 139-152.

BASKIN, C. C. \& J. M. BASKIN. 2014. Seeds: Ecology, Biogeography, and Evolution of Dormancy and Germination. Elsevier Inc., San Diego.

ÇATAV, Ş. S., K. KÜÇÜKAKYÜZ, K. AKBAŞ \& Ç. TAVŞANOĞLU. 2014. Smoke-enhanced seed germination in Mediterranean Lamiaceae. Seed Sci Res. 24: 257-264.

CHEN, S., H. YU, H. LUO, Q. WU, C. LI \& A. STEINMETZ. 2016. Conservation and sustainable use of medicinal plants: problems, progress, and prospects. Chin. Med. 11: 37.

DREW, B. T. \& K. J. SYTSMA. 2013. The South American radiation of Lepechinia (Lamiaceae): phylogenetics, divergence times and evolution of dioecy. Bot. J. Linn. Soc. 171: 171-19.

DUSCHATZKY, C. B., M. L. POSSETTO, M. C. FERNÁNDEZ BELMONTE, M. PEROTTI, C. SCHUFF, C. NÚÑEZ, C. GARCÍA, E. ACOSTA \& E. DAMONTE. 2007. Composición química y actividad antiviral de aceites esenciales de especies autóctonas del centro oeste de Argentina. XVI Simposio Nacional de Química Orgánica, Mar del Plata.

FUNES, G., S. DÍAZ \& P. VENIER. 2009. La temperatura como principal determinante de la germinación en especies del Chaco seco de Argentina. Ecol. Austral 19: 129-138.

GALÍNDEZ, G., G. MALAGRINA, D. CECCATO, T. LEDESMA, L. LINDOW-LÓPEZ \& P. ORTEGABAES. 2015. Dormición física y conservación ex situ de semillas de Amburana cearensis y Myroxylon peruiferum (Fabaceae). Bol. Soc. Argent. Bot. 50: 153-161.

GIORGIS, M. A., R. E. MORERO, A. M. CINGOLANI \& M. CABIDO. 2010. Análisis de la flora medicinal del chaco serrano de la provincia de Córdoba, Argentina. X Simposio Argentino y XII Simposio Latinoamericano de Farmacobotánica, Córdoba.

HONG, T. D. \& R. H. ELLIS. 1996. A protocol to determine seed storage behaviour. IPGRI Technical Bull. 1. International Plant Genetic Resources Institute, Rome.

HUDSON, A. R., D. J. AYRE \& M. K. OOI. 2015. Physical dormancy in a changing climate. Seed Sci. Res. 25: 66-81.

IUCN (International Union for Conservation of Nature). 2007. Species survival commission medicinal plant specialist group. Why conserve and manage medicinal plants? Disponible en: http://www.iucn. org/themes/ssc/sgs/mpsg/main/Why.html [Acceso: 13 octubre 2015].

O'LEARY, N. 2015. Tipificaciones en especies sudamericanas de Hedeoma, Lepechinia y Stachys (Lamiaceae) y nuevo sinónimo en Lepechinia. Bol. Soc. Argent. Bot. 50: 217-220.

PALACIOS, S. M., M. E. MAGGI, C. M. BAZÁN, M. C. CARPINELLA, M. TURCO, A. MUÑOZ, R. A. ALONSO, C. NÚÑEZ, J. J. CANTERO, M. T. DEFAGÓ, C. G. FERRAYOLI \& G. R. VALLADARES. 2007. Screening of Argentinian plants for pesticide activity. Fitoterapia 78: 580-584.

R DEVELOPMENT CORE TEAM. 2014. R: A language and environment for statistical computing. $\mathrm{R}$ foundation for statistical computing, Vienna. Available in: http://www.R-project.org.

SCANDALIARIS, M. \& G. E. BARBOZA. 2013. Caracterización morfohistológica de frutos en especies argentinas de Lepechinia (LamiaceaeNepetoideae-Mentheae). Bonplandia 22: 39-46.

SCHIPPMANN, U., D. J. LEAMAN \& A. B. CUNNINGHAM. 2002. Impact of cultivation and gathering of medicinal plants on biodiversity: Global trends and issues. FAO, Biodiversity and the ecosystem approach in agriculture, forestry and fisheries, Rome, Geneva. 
SERRI, H., A. URBINA, A. CABRERA, T. MILLÁN, E. PASTENE \& J. RUBILAR. 2011. Propagación y Descripción Morfo-Anatómica de Salvia Chilena (Lepechinia chamaedryoides). Bol. Latinoam. Caribe Plant. Med. Aromát. 10: 507-524.

SUSKO, D. J., J. P. MUELLER \& J. F. SPEARS. 2001. An evaluation of methods for breaking seed dormancy in kudzu (Pueraria lobata). Can. J. Bot. 79: 197-203.

VASISHT, K., N. SHARMA, M. KARAN. 2016. Current Perspective in the international trade of medicinal plants material: An update. Curr. Pharm. Des. 22: 4288-4336.

VENABLES, W. N. \& B. D. RIPLEY. 2002. Modern Applied Statistics with $S .4^{\text {th }}$ Ed. Springer, New York. Available in: ISBN 0-387-95457-0.

Recibido el 26 de enero de 2017, aceptado el 27 de marzo de 2017. 